\title{
MECHANISMS OF INSOMNIA FORMATION WITH GASTROESOPHAGEAL REFLUX DISEASE, TAKING INTO ACCOUNT THE PSYCHOSOMATIC STATUS IN YOUNG PEOPLE
}

DOI: 10.36740/WLek202007111

\author{
Aleksey A. Oparin, Iryna 0. Balaklytska, Olga G. Morozova, Anatolii G. Oparin, Ludmila 0. Khomenko \\ KHARKIV MEDICAL ACADEMY OF POSTGRADUATE EDUCATION, KHARKIV, UKRAINE
}

\begin{abstract}
The aim: The work is aimed to study the formation mechanisms of sleep disturbances with GERD based on the determination of melatonin and serotonin indicators in the blood, psychosomatic status and quality of sleep, motor-secretory function of the esophagus and stomach in young people.

Materials and methods: All 63 patients were with non-erosive GERD and insomnia. Among them, 32 with inhibitory personality type and 31 with excitable personality type. The control group is 25 people. Questionnaires were used to determine the quality of sleep, quality of life, state of the nervous system, ultrasound, stomach acidity, level of melatonin and serotonin.

Results: In patients with inhibitory personality type, depressive disorders, lower sleep quality and quality of life were more pronounced, and in patients with excitable type, increased levels of reactive and personal anxiety and pronounced motor-secretory disorders.

Conclusions: Psychosomatic disorders in GERD have a significant impact not only on the quality of sleep, quality of life, the level of melatonin and serotonin, the regulation of the secretory and motor functions of the stomach, but they are one of the links in the pathogenetic mechanism of the formation of both GERD and insomnia with it.
\end{abstract}

KEY WORDS: gastroesophageal reflux disease, insomnia, life quality, melatonin, serotonin

Wiad Lek. 2020;73(7):1365-1369

\section{INTRODUCTION}

Gastroesophageal reflux disease (GERD) is one of the leaders among diseases of the digestive tract. Despite global distribution [1], the incidence rate in different countries varies (from 2.5 in China to 51.2 in Greece) [2]. Herewith, recent studies show that GERD increasingly affects young and able-bodied population [3]. Many studies show that, in addition to age, risk factors for the development of this disease are obesity, hiatal hernia, alcohol consumption and smoking. However, a recent large crossover study showed that a high somatization level according to SSC (somatization scale) is also a common risk factor [4]. Typical symptoms of GERD can affect performance, sleep, nature of nutrition, daily activity, which significantly reduces the patient's life quality [5]. Moreover, sleep disturbance with GERD occurs from 26 to $60 \%$ and has a proven, bi-directional communication [6]. In addition, emotional disturbances, including depression and anxiety, often accompany GERD [7]. It is known that psychological stress can cause insomnia as much as GERD.

Since GERD is diagnosed more often in the adult population, the study of this pathology in young people, in particular students, is rare [8]. On average GERD can be found among students no more than among overall population. However, since it is at this age that the pathogenetic mechanisms of this disease are laid, then with appropriate measures, both therapeutic and prophylactic, one can achieve a favorable course of this disease.
Moreover, to date, the mechanisms of the formation, course, diagnostics and treatment of sleep disturbances in young people, in particular students with GERD, taking into account the psychosomatic status, are not fully understood, which determined the purpose of our study.

\section{THE AIM}

The work is aimed to study the formation mechanisms of sleep disturbances with GERD based on the determination of melatonin and serotonin indicators in the blood, psychosomatic status and quality of sleep, motor-secretory function of the esophagus and stomach in young people.

\section{MATERIALS AND METHODS}

We examined 63 students hospitalized in the gastroenterological department of the Municipal Non-Commercial Enterprise "City Student Hospital", Kharkiv, Ukraine. All patients with non-erosive GERD were divided into two clinically homogeneous groups. The first group included 32 patients with GERD and insomnia and inhibitory personality type (15 men and 17 women) whose average age was 20.5 years old. The second group - GERD with insomnia and excitable personality type -31 patients ( 15 men and 16 women) whose average age was 20.1 years old. The control group consisted of 25 healthy patients ( 12 men 
and 13 women) whose age was 20.2 years old. All patients had a non-erosive GERD form. The case history of GERD ranged from 1 to 5 years. An average is of 3.4 years. GERD was diagnosed according to ICD-10 and the recommendations of the 2006 Montreal Consensus. The criteria for the exclusion of students from the observation groups were the presence of concomitant pathology of other organs and systems and inflated self-esteem of psychosomatic status according to any of the questionnaire scales, which, in conformity with instructions, required a psychotherapist consultation.

Prior to the study, all sick and healthy students of the control group were informed of its aims. A voluntary consent for the study was obtained. All procedures performed in the study comply with the Declaration of Helsinki.

In order to study sleep disturbances in patients of both groups, the Pittsburgh Sleep Quality Index (PSQI) was used. The following components of sleep were evaluated: subjective sleep quality, sleep latency, sleep duration, sleep efficiency, sleep disturbance, use of sleep medication, daytime dysfunction. Each component was evaluated from 0 to 3 , where 0 indicates the absence of sleep disturbances, and 3 indicates serious disturbances. The sum of the scales results varies from 0 to 21. A higher score indicates the worst sleep quality.

The presence and severity of depression were determined using the PHQ-9 questionnaire (Patient Health Questionnaire). This questionnaire consists of nine questions. Each question has four possible answers and assessment from 0 to 3 , depending on the problem frequency. If the total number of scores is from 0 to 4 , this means the absence of depression, from 5 to 9 - mild depression, from 10 to 14 - moderate one, from 15 to 19 - moderately severe and from 20 to 27 - severe depression.

The psychosomatic condition of patients was assessed according to the Spielberger-Khanin scale to determine personal and situational anxiety. When analyzing the results, the total indicator for each of the subscales can be in the range from 20 to 80 scores. Moreover, the higher the final indicator, the higher the level of anxiety (situational or personal). When interpreting the indicators, the following anxiety assessments are used: up to 30 scores - low, 31-44 scores - moderate; 45 and more - high.

Life quality was assessed using the unified SF-36 questionnaire, which includes assessment of the physical health scale: role-physical functioning (RP), physical functioning $(\mathrm{PF})$, bodily pain $(\mathrm{BP})$, general health $(\mathrm{GH})$, and the mental health scale: role-emotional functioning (RF), vitality scale (VT), mental health $(\mathrm{MH})$, and social functioning (SF). Indicators of each scale ranged from 0 (the lowest level) to 100 scores (full health level).

The acidity of the gastric juice was determined by intragastric $\mathrm{pH}$-metery using IKZh-2 apparatus (produced in Ukraine).

Motor-evacuatory function of the esophagus and stomach was identified using an ultrasound examination conducted on ALOKA SSD-650 machine (produced in Japan) and Ultima pro-30 machine (produced in Ukraine) - in the patient's left side and on the back; in epigastric region and projections at esophageal hiatus; fasted and 5, 10 and 1 minutes after drinking $0.5 \mathrm{l}$ of liquid. The study measured the thickness of the esophagus wall, the esophagus width in the lower third, the diameter of esophageal hiatus, the presence of fluid regurgitation, and its duration.

Melatonin and serotonin in blood serum were determined by immunoenzyme method using a standard IBL set of reagents (produced in Germany). Since melatonin has daily fluctuations, blood sampling was performed at the same time of the day for all subjects (7.00 in the morning).

To process statistical data, we used Microsoft Office Excel 2007 and the statistical data processing system Statistica 6.0. Under normal distribution, Student's t-test was used; in case of deviation from the normal distribution, nonparametric methods were used. The mean value (M), Student's t-test was assessed. The differences were considered significant at $p<0.05$. The results were presented as $M \pm m$, where $\mathrm{M}$ is the median and $\mathrm{m}$ is the standard deviation.

\section{RESULTS}

In the course of our studies, we found that in the clinic picture in most patients (76.8\%) among both excitable and inhibitory ones, heartburn was the guiding symptom.

When studying the sleep quality index in GERD patients with insomnia according to the Pittsburgh Questionnaire (PSQI), the following results were revealed: subjective sleep quality in group 1 was 1.86 , in group $2-2.47$, in the control group -0.53 . The index of sleep latency in group 1 was 2.4 , in group $2-2.7$, in the control group -0.32 . The sleep duration indicator in group 1 was 0.95 , in group $2-$ 1.46 , in the control group -0.3 . The sleep efficiency index in patients of group 1 was 1.05, in group $2-1.54$, in the control group -0.24 . The rate of sleep disturbance in group 1 was 1.16 , in group $2-1.52$, in the control group -0.25 . None of the patients used sleeping pills, so this indicator for all was 0 . The daytime dysfunction indicator in group 1 was 1.27 , in group $2-2.01$, in the control group - 0.15 . The average total score for group 1 was 8.72 , for group 2 11.7 , for the control group -1.8 .

Thus, sleep disturbance was observed in patients of both groups, but insomnia $(\mathrm{p}<0.05)$ was more pronounced in patients of group 2 as compared not only to the control group, but also to the patients of group 1 .

When studying the presence and severity of depression in GERD patients with insomnia according to PHQ-9 (Patient Health Questionnaire), the following results were revealed. Moderate depression was more pronounced in patients of group $2-82.12 \%$ than in group $1-67.32 \%$, and in the control group it was absent $(\mathrm{p}<0.05)$. Mild depression in group 1 was $20.63 \%$, in group $2-13.34 \%$, in the control group $-3.3 \%(\mathrm{p}<0.05)$. Depression was absent in $96.7 \%$ in the control group, in group $1-12.05 \%$, in group $2-4.54 \%(\mathrm{p}<0.05)$.

When determining the psychosomatic status in GERD patients with insomnia, as well as in people of the control group, the following results were obtained. Personal 
anxiety in the excitable type is $49.1 \pm 1.4$, in the inhibitory type $-36.1 \pm 1.1$, and in individuals in the control group $22.4 \pm 1.2(\mathrm{p}>0.05)$. Reactive anxiety in the excitable type is $46.8 \pm 1.9$, in the inhibitory $-36.9 \pm 1.2$, and in the control

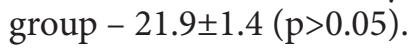

With a comprehensive assessment of the psychosomatic status of patients, the following conclusions can be drawn: in the inhibitory type, the depression indicators come to the fore, and in the excitable one increased indicators of personal and reactive anxiety are more pronounced.

The life quality indicators for GERD patients with insomnia, as well as for individuals of the control group according to the unified SF-36 questionnaire, are as follows: physical functioning in group 1 was $43.7 \pm 3.8$, in group 2 - 40.3 \pm 3.9 , in the control group - 84.5 \pm 4.0 ; role-physical functioning in group 1 was $27.0 \pm 3.3$, in group $2-36.7 \pm 4.0$, in the control group $-71.4 \pm 3.0$; the bodily pain in group 1 was $41.2 \pm 3.6$, in group $2-23.1 \pm 3.8$, in the control group $76.3 \pm 4.0$; general health in group 1 was $35.5 \pm 3.9$, in group $2-32.2$, in the control group $-74.9 \pm 4.1$; vitality in group 1 was $45.5 \pm 3.2$, in group $2-31.5 \pm 4.2$, in the control group $-80.5 \pm 3.2$; social functioning in group 1 was $45.2 \pm 3.5$, in group $2-43.6 \pm 3.3$, in the control group $-81.4 \pm 4.4$; role-emotional functioning in group 1 was $48.3 \pm 3.1$, in group $2-31.7 \pm 3.6$, in the control group - 78.6 \pm 3.5 ; mental health in group 1 was $44.5 \pm 3.3$, in group $2-30.5 \pm 2.7$, in the control group $-75.4 \pm 3.7$.

It was found that in patients of both groups, the life quality indicators were significantly reduced according to all scales of the SF-36 questionnaire as compared to the control group and the difference was statistically significant $(\mathrm{p}<0.001)$. Moreover, in patients of group 2, indicators of vitality, bodily pain, role-emotional functioning and mental health were significantly $(\mathrm{p}<0.001)$ lower than in patients of group 1 . At the same time the indicator of role-physical functioning was lower $(\mathrm{p}<0.001)$ in patients 1. The indicators of general health, physical and social functioning were lower in patients of group 2 as compared to group 1 , but the difference was statistically insignificant $(\mathrm{p}>0.05)$.

The indicators of $\mathrm{pH}$-metery of the stomach body in patients of group 1 are $0.91 \pm 0.25$, in group $2-1.34 \pm 0.05$, in the control group - $1.61 \pm 0.1$; in the antrum, group 1 had $5.9 \pm 0.15$, group 2 had $4.7 \pm 0.17$, and control group had $7.21 \pm 0.12$.

In patients with excitable type, the $\mathrm{pH}$-metery level in the body was statistically significantly lower $(p<0.05)$ than in the group of patients with the prevalence of inhibitory personality traits in the psychosomatic status and in the control group. The neutralizing stomach activity in both groups was lower than that of the control group $(\mathrm{p}<0.05)$.

When examining motor-evacuatory function of the esophagus and stomach in GERD patients with insomnia, as well as in the control group, the following results were revealed: the esophagus width in the lower third in patients of group 1 was $2.63 \pm 2.8 \mathrm{~cm}$., in patients of group $2-2.57 \pm 0.27 \mathrm{~cm}$., in the control group $-2.11 \pm 0.1 \mathrm{~cm}$.; the diameter of esophageal hiatus in patients of group 1 was $1.93 \pm 0.27 \mathrm{~cm}$., in patients of group $2-1.83 \pm 0.25 \mathrm{~cm}$., in the control group - $1.51 \pm 0.12 \mathrm{~cm}$.; the presence and duration of gastroesophageal reflux in patients of group 1 was 9 minutes \pm 1.3 , in patients of group $2-7$ minutes \pm 1.3 , in the control group - 5 minutes \pm 1.1 ; the thickness of the esophagus wall in patients of group 1 was $0.46 \pm 0.05$ $\mathrm{cm}$., in patients of group $2-0.40 \pm 0.03 \mathrm{~cm}$., in the control group $-0.31 \pm 0.07 \mathrm{~cm}$.

Thus, it was found that all indicators were significantly greater in patients of group 1 than in group $2(\mathrm{p}<0.05)$.

When studying the melatonin and serotonin indicators in blood serum in GERD patients with insomnia, as well as in the control group, the following results were revealed: melatonin in patients of group 1 was $14.4 \pm 1.5 \mathrm{pg} / \mathrm{ml}$, in patients of group $2-7.5 \pm 0.74 \mathrm{pg} / \mathrm{ml}$, in the control group $-22.3 \pm 1.8 \mathrm{pg} / \mathrm{ml}$. Serotonin in patients of group 1 was $81.7 \pm 5.3 \mathrm{pg} / \mathrm{ml}$, in patients of group $2-118.3 \pm 7.2 \mathrm{pg} / \mathrm{ml}$, in the control group $-34.7 \pm 2.7 \mathrm{pg} / \mathrm{ml}$.

Thus, in patients with inhibitory type, we observed the lowest melatonin and the highest serotonin indicators.

\section{DISCUSSION}

Sleep disturbances with GERD can be associated with several reasons [9]. The main one is the presence of night reflux and night heartburn [10]. The following causes of sleep disturbance with GERD are the presence of anxiety and depression. Okuyama et al. show that patients with GERD have more frequent manifestations of anxiety and depression as compared to the control group [11]. These data are consonant with our study, since increased anxiety and depression were significantly higher in patients of both groups. But we have also shown the difference between these indicators in inhibitory and excitable young patients.

In a study of medical students in Iran who suffered from GERD, $47.2 \%$ of them had anxiety, $22.5 \%$ had depression, $36 \%$ had somatic symptoms, and $36 \%$ had social dysfunction [8]. In our study, the mentioned indicators are higher and this may be due to the presence of insomnia in our patients, which exacerbates the course of GERD; it has a direct influence on the indicators of psychosomatic status of patients.

The relationship between GERD and psychosomatic disorders has been shown in many studies [12]. A study by Ping $\mathrm{Li}$ et al. showed that a combination of several atypical GERD symptoms increases the risk of psychological disturbances [13].

It is known that patients with depression have a higher severity of various symptoms and are more likely to describe the symptoms in a negative light [11]. In our study, the patients with inhibitory type had a higher level of depressive disorders and life quality. Their sleep quality was significantly lower not only in comparison with the control group, but also with the excitable type.

Our observations showed a significant decrease in the quality of life in patients of both groups. Persistent GERD symptoms cause a decrease in life quality and social activity [14]. According to large studies, GERD patients with sleep 
disturbances had lower life quality indicators associated with health as compared to GERD patients without sleep disturbances [15]. The physical component of life quality was particularly reduced [16]. At the same time, sleep disturbances significantly reduce not only the life quality but also the effectiveness of leisure and work productivity, which is also an important economic problem.

A significant number of studies determine sleep quality using the PSQI questionnaire. Sleep disturbances in this questionnaire were more common in patients with GERD than in the control group. 52\% experienced sleep disturbances, with PSQI indicators of more than 5.5 [7]. Our patients in both groups had PSQI of more than 6.2.

The presence of stress in the life of students is not in doubt. At least $82 \%$ of the 2246 studied students suffered from physical or mental distress [17].

Herewith a number of studies have shown that patients who are under chronic stress and in state of anxiety may experience esophageal hypersensitivity, which is included in the pathogenesis of GERD [11].

At the same time, prolonged and intense exposure to psychological stressors leads to overload and depletion of the central nervous system, resulting in disorder of its regulatory function, which, together with functional disorders of organs and systems, creates the prerequisites for the development of psychosomatic pathology [18].

Taking into account our results [19] and the results of other studies [8], we can talk about a close relationship between psychosomatic disorders and GERD.

Stress can increase or trigger the formation of GERD. At the same time, the perception of GERD symptoms itself is enhanced much more than the actual presence of the symptoms severity. A number of studies show that stress-reducing GERD therapy reduces the perception of symptoms and reduces the effect of acid on the esophagus, which in turn confirms the interaction of the brain with the digestive tract [20].

Numerous studies show that stress changes the stomach acidity [21]. In our study, we also obtained the characteristics of acidity in patients with GERD, depending on the personality type and psychosomatic disorders.

Melatonin is a hormone that controls sleep and gastrointestinal motility. Low melatonin indicators can cause both GERD and insomnia [22,23]. In our study, patients in both groups also had low melatonin indicators. Serotonin and melatonin are in constant opposition and create a balance in the body. The motility of the digestive tract is a clear illustration of this process [24].

\section{CONCLUSIONS}

1. It was found that students with GERD have marked disturbances of sleep, quality of life, motor-secretory disorders, and psychosomatic disorders. Moreover, in patients with inhibitory type in psychosomatic status, depressive disorders, lower sleep quality, and life quality come to the fore, while in patients with excitable type - increase in the level of reactive and personal anxiety and pronounced motor-secretory disorders.
2. In patients of both groups, a significant decrease in the level of melatonin is determined, with a simultaneous increase in the level of serotonin. In patients with inhibitory personality type, these differences are more significant as compared to the group of GERD patients with excitable personality type.

3. A clear correlation was established between the degree of decrease in melatonin level, increased serotonin, sleep quality indicators, characteristics of psychosomatic disorders, life quality, and motor-secretory disorders of the esophagus and stomach.

4. The data obtained indicate that psychosomatic disorders with GERD significantly affect only the sleep quality, life quality, the level of melatonin and serotonin, the regulation of the secretory and motor functions of the stomach, but they are also one of the links of the pathogenetic mechanism for the formation of both GERD and insomnia.

\section{REFERENCES}

1. Gyawali C.P., Fass R. Management of Gastroesophageal Reflux Disease. Gastroenterology. 2018;154(2):302-318.

2. Eusebi L.H., Ratnakumaran R., Yuan Y. et al. Global prevalence of, and risk factors for, gastro-oesophageal reflux symptoms: A meta-analysis. Gut 2018;67(3):1-11.

3. Kim G.H. It Is Time to Meet the Challenges of the Changing Epidemiology of Gastroesophageal Reflux Disease. J Neurogastroenterol Motil. 2018;24(4):507-509.

4. Kim S.Y., Jung H.K., Lim J. et al. Gender Specific Differences in Prevalence and Risk Factors for Gastro-Esophageal Reflux Disease. J Korean Med Sci. 2019;34(21):e 158.

5. GuanX.L.,Wang H. Quality of lifescales for patients with gastroesophageal reflux disease: A literature review.Int J Nurs Sci. 2015;2:110-114.

6. Okuyama M., Takaishi 0., Nakahara K. et al. Associations among gastroesophageal reflux disease, psychological stress, and sleep disturbances in Japanese adults. Scand J Gastroenterol. 2017;52(1):44-49.

7. Kim J.Y., Kim N., Seo P.J. et al. Association of sleep dysfunction and emotional status with gastroesophageal reflux disease in Korea. J Neurogastroenterol Motil. 2013;19(3):344-353.

8. Bordbar G., Bolandnazar N.S. Gastroesophageal reflux disease (GERD): prevalence and association with Psychological Disorders among medical sciences students. Int J PharmTech Res. 2015;8(7):120-130.

9. Lindam A., Ness-Jensen E., Jansson C. et al. Gastroesophageal Reflux and Sleep Disturbances: A Bidirectional Association in a Population-Based Cohort Study, The HUNT Study. Sleep. 2016;39(7):1421-1427.

10. Lim K.G., Morgenthaler T.I., Katzka D.A. Sleep and Nocturnal Gastroesophageal Reflux: An Update. Chest. 2018;154(4):963-971.

11. Okuyama M., Takaishi 0., Nakahara K. et al. Associations among gastroesophageal reflux disease, psychological stress, and sleep disturbances in Japanese adults. Scand J Gastroenterol. 2017;52(1):44-49.

12. Lim C.H., Choi M.G., Baeg M.K. et al. Symptom characteristics and psychosomatic profiles in different spectrum of gastroesophageal reflux disease. Gut Liver. 2014;8(2):165-169.

13. Li P., Wang F., Ji G.Z. et al. The psychological results of 438 patients with persisting GERD symptoms by Symptom Checklist 90-Revised (SCL-90-R) questionnaire. Medicine (Baltimore). 2018;97(5):e9783. doi: 10.1097/ MD.0000000000009783. 
14. You Z.H., Perng C.L., Hu L.Y. et al. Risk of psychiatric disorders following gastroesophageal reflux disease: A nationwide population-based cohort study. Eur J Intern Med. 2015;26(7):534-539.

15. Iwakura N., Fujiwara Y., Shiba M. et al. Characteristics of Sleep Disturbances in Patients with Gastroesophageal Reflux Disease. Intern Med. 2016;55(12):1511-1517.

16. Oparin A., Vnukova A. The role of the endothelial dysfunction in the mechanism of formation of gastroesophageal reflux disease in patients with ischemic heart disease. Acta Clin Croat. 2017; 56: 635-639.

17. Dyrbye L.N., Harper W., Durning S.J. et al. Patterns of distress in US medical students. Med Teach. 2011;33(10):834-839.

18. Oparin A., Bezjazichnaya N. Implementation mechanisms of psychosomatic disorders in gastroesophageal reflux disease with concomitant chronic obstructive pulmonary disease. Med Jad. 2016;46(3-4):125-132.

19. Oparin A., Kornienko D. Formation process of motor-evacuatory disorders in patients with gastroesophageal reflux disease and concomitant obesity. Gastroent Hepatol. 2017;71(2):145-149.

20. Jansson C., Wallander M.A., Johansson S. et al. Stressful psychosocial factors and symptoms of gastroesophageal reflux disease: a populationbased study in Norway. Scand J Gastroenterol. 2010;45(1):21-29.

21. Sandhu D.S., Fass R. Stress and gastroesophageal reflux disease. Proc Shevchenko Sci Soc Med Sci. 2018;54(2):10-15.

22. Kandil T.S., Mousa A.A., El-Gendy A.A. et al. The potential therapeutic effect of melatonin in gastroesophageal reflux disease. $B M C$ Gastroenterol. 2010;10(7).

23. Oliveira-Torres J.D.F. Which is the best choice for gastroesophageal disorders: Melatonin or proton pump inhibitors? World J of Gastrointest Pharmacol and Ther.2010;1(5):102-106.

24. Thor P.J., Krolczyk G., Gil K. et al. Melatonin and serotonin effects on gastrointestinal motility. J Physiol Pharmacol. 2007;58(6):97-105.
This article is written according to the research project of Department of Therapy, Rheumatology, and Clinical Pharmacology of Kharkiv Medical Academy of Postgraduate Education. The title of project is "The pathogenetical mechanisms of comorbidity formation in students with GERD, and the correction of these mechanisms" (state registration number 0110U002441).

\section{ORCID and contributionship:}

Aleksey A. Oparin: 0000-0003-1493-9353 ${ }^{A, C}$

Iryna O. Balaklytska: 0000-0003-3879-8997 B,D

Olga G. Morozova: 0000-0002-3088-624X ${ }^{F}$

Anatolii G. Oparin: 0000-0002-5501-3891 ${ }^{\mathrm{A}}$

Ludmila O. Khomenko: 0000-0003-0275-532X ${ }^{E}$

\section{Conflict of interest:}

The Authors declare no conflict of interest.

\section{CORRESPONDING AUTHOR Iryna 0. Balaklytska}

Kharkiv Medical Academy of Postgraduate Education

58 Amosova st., 61176 Kharkiv, Ukraine

tel: +38066 1620331

e-mail: balaklytska@karazin.ua

Received: 10.10 .2019

Accepted: 22.05 .2020

A - Work concept and design, B - Data collection and analysis, C - Responsibility for statistical analysis, D-Writing the article, $\mathbf{E}$-Critical review, $\mathbf{F}$ - Final approval of the article 